\title{
Democracia y participación ciudadana en el Ecuador. ¿Un espejismo?
}

\section{Democracy and citizen participation in Ecuador. A mirage?}

\author{
Marco Vinicio Carrión Torres ${ }^{1}$, Doris Alexandra Contento Armijos ${ }^{2}$ \& Denise Liliana \\ Pazmiño Garzón ${ }^{3}$
}

Recibido: 01-07-2019 / Revisado: 15-07-2019 /Aceptado: 04-08-2019/ Publicado: 10-09-2019

\begin{abstract}
.
DOI: https://doi.org/10.33262/cienciadigital.v3i3.4..829

This research work determines the conceptualization of citizen participation in Latin America and its appearance in Ecuador, as well as its normative and legal structure of legitimacy that allows it to operate, in addition to it seeks to determine the participation spaces that the Ecuadorian has as an actor fundamental in the design of public policies and retro feeder of public management in the central state bodies and Decentralized Autonomous Governments, for this two sequences were established: the first, a historical journey of participatory democracy with its experiences in Latin America and its affinity in Ecuador, the second, manages to determine the mechanisms, spaces and institutions of citizen participation that Ecuadorian legislation has and that approximate State-society relations; This analysis ends with the understanding of the state of citizen participation in Ecuador from the standpoint of conceptualization and legitimacy, especially since the drafting of the 2008 constitution becomes relevant and revalues the rights of the citizen in Ecuador and determines the transversality of citizen participation in national public management.
\end{abstract}

Keywords: Participatory democracy, representative democracy, citizen participation, institutionalization.

\section{Resumen}

El presente trabajo de investigación determina la conceptualización de la participación ciudadana en Latinoamérica y su aparición en el Ecuador, así como su estructura

\footnotetext{
${ }^{1}$ Universidad de las Fuerzas Armadas, Riobamba, Ecuador. mvct1980@hotmail.com

${ }^{2}$ Magíster en Gestión Pública. Servicios Profesionales. alecontento@gmail.com

${ }^{3}$ Escuela Superior Politécnica de Chimborazo, Facultad de Administración de Empresas. Riobamba, Ecuador. denise.pazmino@espoch.edu.ec
} 
normativa y jurídica de legitimación que la permite accionar, además de ello busca determinar los espacios de participación que tiene el ecuatoriano como actor fundamental en el diseño de políticas públicas y retro alimentador de la gestión pública en los entes del estado central y Gobiernos Autónomos Descentralizados, para ello se establecieron dos secuencias: la primera, un recorrido histórico de la democracia participativa con sus experiencias en Latinoamérica y su afincamiento en el Ecuador, la segunda, consigue determinar los mecanismos, espacios e instituciones de participación ciudadana con los que cuenta la legislación ecuatoriana y que aproximan las relaciones Estado-sociedad; terminando este análisis de consigue comprender el estado de la participación ciudadana en el Ecuador desde la óptica de conceptualización y legitimidad, sobre todo desde la redacción de la constitución del 2008 cobra relevancia y revaloriza los derechos del ciudadano en el Ecuador y se determina la transversalidad de la participación ciudadana en la gestión pública nacional.

Palabras claves: Democracia participativa, democracia representativa, participación ciudadana, institucionalización.

\section{Desarrollo}

La relación Estado-sociedad en los países a través del tiempo ha tenido, modificaciones sustanciales, la entrega del poder por parte del ciudadano hacia los entes políticos de representación marcó el rumbo en distintas vías para un beligerante desarrollo general. El tamaño del Estado identificaba el modelo político de una ideología partidista al momento de gobernar, así como los tipos de democracia que se utilizaron para determinar los asuntos públicos. Para este estudio se identifican dos tipologías: la democracia representativa y la democracia participativa; la primera en resumen es entendida como el sistema en que el ciudadano tiene la facultad para entregar por medio del voto su porcentaje de poder a otro con capacidad de representarlo y la segunda que se " basa en la idea que los ciudadanos deben participar directamente en las decisiones politicas y no solo como pretende la democracia representativa en la eleccion de los decisores políticos" (de Sousa Santos, 2003:7), por lo tanto la democracia participativa por naturaleza crea nuevos espacios para la deliberación y toma de decisiones en conjunto, pero hay ahí una dicotomía por resolver, ¿quién es el encargado de crear estos espacios de participación?, ¿es el Estado el llamado en crearlos? o ¿es la sociedad desde sus bases o movimientos sociales los encargados de hacerlo?, más adelante se realizará un análisis más profundo de las dos democracias brevemente descritas.

En el año 2008 con la vigencia de la nueva versión de la Constitución ecuatoriana, se rescataron un sinnúmero de adhesiones en el campo de los derechos de la ciudadanía, entre ellos, los derechos de participación contenidos en el artículo 61 del cuerpo legal, con esto se busca una profundización democrática del Ecuador, además se incorpora como necesidad e 
innovación, la creación de la Función ${ }^{4}$ de Transparencia y Control Social, que "se justifica con la finalidad de impulsar el control y transparencia del sector público y del privado que desarrolle sus actividades de interés público, el impulso de la participación ciudadana, y el permanente combate de la corrupción" (Cañas \& Birck, 2011:77), esta función a su vez está compuesta por cinco entidades; entre ellas está el Consejo de Participación Ciudadana y Control Social (CPCCS), parte importante en el desarrollo del presente trabajo de investigación.

El objetivo general de este estudio es determinar si existe en el Ecuador e ejercicio de una participación ciudadana legítima y espontánea, que se enmarque en los instrumentos de la democracia participativa y se involucre en la toma de decisiones, planificación y cogestión de los asuntos públicos.

Como objetivos específicos de este trabajo académico-investigativo están:

1. Comprender las conceptualizaciones y aportes de varios autores sobre la democracia participativa, democracia representativa y participación ciudadana además de sus condiciones históricas y sociales sobre las cuales surge en Latinoamérica y en Ecuador específicamente; así se podrá comprender la valía de esta evolución democrática e identificar el papel que debe desempeñar el ciudadano en el Estado.

2. Establecer los mecanismos, dispositivos e instituciones previstos en la Constitución de la República del Ecuador del año 2008 y en la legislación vigente que fomentan la democracia participativa y tratar a profundidad el bloque de la participación ciudadana en la gestión pública, esto ayudará a visualizar el espectro jurídico de la cuestión.

Para el desarrollo de este documento se presenta 2 partes, siendo al final el propósito de este estudio, aportar desde la óptica académica la influencia que tienen los actores de la participación ciudadana en una joven democracia participativa y sobre la gestión de la política pública actual. Entonces el método de esta investigación será cualitativo, soportada en fuentes primarias de carácter bibliográfica, el tipo de investigación sera documental puesto que "consiste en un análisis de la información escrita sobre un determinado tema con el propósito de establecer relaciones, diferencias, etapas, posturas (...)" (Bernal, 2006:110); además se sintetizará información cuantitativa obtenida de fuentes secundarias con el fin de complementar la investigación.

\section{I) Democracia Participativa hacia el refuerzo de la Representación}

El concepto de democracia ha sido revestida por temporalidades y condiciones evolutivas, se la considera como una utopía social sin fin, al remontarse a los orígenes la palabra democracia significa "gobierno del pueblo", es decir que existe democracia cuando los

\footnotetext{
${ }^{4}$ Cabe destacar que en la Constitución previa a la del 2008 existían únicamente 3 poderes: Ejecutivo, Legislativo y Judicial, en los cuales de dejaba de lado la participación de la ciudadanía la que al fin y al cabo da su poder como mandantes a sus representantes para gobernarlos. Pongo el texto ya que se debe recalcar la relevancia del drástico cambio de la Constitución frente a la inclusión del ciudadano en la toma aunque sea en documentos sobre su rumbo y no dejarlo en manos de los poderes ya maneados por los políticos de turno.
} 
integrantes de una sociedad toman las decisiones en base a sus pensamientos y a sus consensos, "los principios de la democracia en su versión republicana clásica son la igualdad , el pluralismo político y la deliberación política, bajo trasfondo de la libertad." (Curnill Grau, 1997:72), "la deliberación política", es un principio que debe asumir el ciudadano en las diversas formas de organización social al momento de tratar asuntos públicos, la excesiva entrega de la voluntad ciudadana a la representación convierte en implicita las decisiones del representado, trayendo consigo presunciones y equivocaciones en la toma de decisiones; al anularse el momento de la deliberación se corre mucho mas riesgo que poderes externos quieran tutelar el desempeño democrático; sin embargo tambien hay que reconocer que para que exista una deliberación eficaz los ciudadanos deben estar preparados responsablemente en los diversos temas que le atañen.

Para entender el surgimiento de la democracia participativa es necesario conocer primero que la democracia representativa, por sí sola estuvo consolidada por casi dos siglos en el viejo continente y en América del Norte, tal fue el impulso a la representación que "partir del Consenso de Washington $^{5}$, la democracia representativa empezó a ser recomendada universalmente e incluso impuesto como parte integrante de los programas de ajuste estructural del FMI o como condición para acudir a préstamos de los países centrales del Banco Mundial y otras agencias multilaterales" (de Sousa Santos, 2003:7), posteriormente a razón de la evolución de la sociedad empezaron a realizarse transformaciones democráticas paulatinamente primero en el sur de Europa (España y Portugal) hasta llegar a algunos países de Latinoamérica, empezándose en Brasil con el Presupuesto Participativo de Porto Alegre a fines de los ochenta, más tarde "a lo largo de la década de los noventa tal impulso participativo provino en lo fundamental del acceso de diversos partidos de izquierda y movimientos populares al poder local, tanto a nivel urbano como rural, el Partido de los Trabajadores en Brasil, el Frente amplio Uruguayo, la Causa R en Venezuela, Gobiernos indígenas participativos, entre otros" (Ramirez \& Welp, 2011:11).

Ahora es necesario entender las diferencias entre democracia representativa y democracia participativa en cuanto a la primera "La representación es una figura originaria del derecho privado, mediante la cual una persona llamada representante asume la voluntad de otra incapaz, llamada representado" (Noguera Fernandez, 2008:135); Rousseau ya tenía reparos frente a la democracia representativa, no admitía que el poder del pueblo se pueda delegar totalmente de esa manera. Según Curnill (1997), la representación tiene tres problemas base: el primero, atrae inequidades en las tareas distributivas del Estado y en la afirmación de

\footnotetext{
${ }^{5}$ Se lo considera como un conjunto de lineamientos macroeconómicos de línea neoliberal aplicada en América Latina en la década de los noventas que pregonaba un modelo económico abierto y estable. (según sus creadores) por un lado, la apertura económica consistía principalmente en una serie de medidas que permitieran y facilitaran el comercio internacional entre los países en vías de desarrollo y los desarrollados, con la finalidad de impulsar el crecimiento económico de la región. Por otra parte, la disciplina macroeconómica proporcionaría la estabilidad económica a partir del control de las finanzas públicas del Estado, para lograr efectos económicos, políticos y sociales positivos.
} 
derechos sociales; el segundo, la probable falta de democratización de las instituciones de representación, parlamentos y partidos políticos que requieren ser impulsadas y complementadas por la sociedad; y tercero, la recuperación de las funciones políticas de la sociedad para presionar sobre la democratización de la representación política y pluralizar el proceso y contenidos de las políticas del Estado (71). La democracia participativa debe ser considerada como complemento de la democracia representativa, no pretende ser un reemplazo, sus instrumentos o mecanismos diseñados se constituyen en espacios participativos que apoyan a perfeccionar y profundizar a la democracia, permiten que los ciudadanos observen, interactuen y se reunan físicamente, acciones que contribuyen a sentirse parte de una decisión y generan seguridad y confianza personal, ésta complementariedad según de Sousa Santos (2003) apoya "a la redistribución de la riqueza, la protección social y a obtener mayor transparencia del poder político".(7)

En cuanto a las condiciones sociales para el surgimiento de la democracia participativa, ésta emerge a manera general por la constante lucha de los sectores marginados, que buscaban reivindicaciones de derechos, es decir uno de los motivos de la aparición de la democracia participativa es la exclusión social y otro el cansancio colectivo de constantes actos de corrupción que ven mermados los recursos públicos a beneficio de elites de poder.

En América Latina en los años sesenta empiezan los primeros pasos de la participación ciudadana en las políticas públicas, con la conformación de los primeros órganos consultivos dedicados a temas económicos de interés nacional y que involucraban a empresarios por el tema de sustitución de importaciones. Más tarde por los años ochenta cuando la mayoría de países de América Latina funcionaban con políticas neoliberales que buscaban un Estado mínimo, aparecen con frecuencia mecanismos de participación ciudadana, los Estados autoritarios fueron también desapareciendo y en el campo económico internacional abrieron las fronteras hacia la globalización e internacionalización de los mercados. La descentralización de los servicios sociales hacia las organizaciones y entidades sociales en el territorio, también fue un aporte instrumental para el desarrollo de la participación ciudadana además fueron apareciendo los primeros espacios legítimos y naturales de participación ciudadana en los municipios en las conocidas audiencias públicas. Sin embargo el devenir de la participación ciudadana en algunos de los países de América Latina no tuvo una trascendencia marcada, intentos esporádicos y fugaces como en el Perú las Asambleas Regionales tuvieron poca vida, asimismo en Chile, un régimen autoritario que instauró los Consejos de Desarrollo Comunal integrado por representantes de organizaciones económicas y sociales también fracasó.

La Participación Ciudadana al pasar de los años fue tomando fuerza e importancia dentro del andamiaje político de los países, esto gracias al incremento en la frecuencia de muestras de participación social. A nivel Internacional la Carta Iberoamericana de Participación Ciudadana en la Gestión Pública declarada en Lisboa en el 2009 menciona que la participación ciudadana permite defender y exigir derechos y además se convierte en un 
medio para la transformación social, tiene características de inclusión hacia los pueblos indígenas y un enfoque de género, pero sobre todo la participación ciudadana se tiene que orientar al principio de corresponsabilidad social (CLAD, 2009:2), es decir al mismo tiempo que exige derechos la participación ciudadana también imprime deberes, característica de una ciudadanía ejemplar, el problema se presenta cuando el habitante de un territorio ejerce o no sus derechos o no cumple con sus deberes, en este sentido la democracia participativa solo podrá ser nutrida por una participación ciudadana interesada, formada y permanentemente activa.

Al llegar en el análisis al Ecuador, los inicios de la participación ciudadana se la detecta en la década de los noventa en la cual ocurrieron importantes acciones sociales, considero evidenciada por dos lados: el lado jurídico institucional y por el lado de los movimientos y organizaciones sociales; en esta década por el lado jurídico institucional se aprobó por la representación parlamentaria la Ley Orgánica de Transparencia y Acceso a la información LOTAIP y la conformación de la Comisión de Control Cívico de la Corrupción CCCC, ambas fueron producto de presiones sociales y descontentos ciudadanos para controlar las acciones de los representantes y detectar actos de corrupción; y por el otro lado sin duda el más representativo de ese decenio es el movimiento indígena que luego de muchos años de sumisión lograron organizarse y se plantaron en la sociedad ecuatoriana como parte social activa en busca de su reconocimiento y reivindicación de sus culturas. En el gobierno del Dr. Rodrigo Borja Cevallos, se produce el primer levantamiento indígena en donde se obtuvo varias conquistas como la inclusión de la educación bilingüe y el seguro social campesino entre otras. La exclusión de estos pueblos provocó a que se organizarán y buscarán protagonismo desde la esfera social, teniendo en cuenta la democracia comunitaria fueron incrementando su poder organizativo a través de sus confederaciones este movimiento posteriormente incursionó en palestra política con la conformación del partido político Pachacutik y ha logrado en base a sus nacionalidades ganar elecciones locales y provinciales.

En años posteriores 1996- 2006 se produce la mayor crisis democrática que ha vivido el Ecuador con presidentes de la república derrocados, inicialmente por shocks económicos políticos como incremento de la pobreza y la deuda externa, el feriado bancario, la dolarización, violaciones constantes de la constitución, pero principalmente el descontento ciudadano se da por ofertas de campaña no cumplidas, cambios de discurso y desarmes de alianzas políticas una vez que el presidente llega al poder; además de los altos niveles de corrupción detectados, estas frustraciones se volvieron repetitivas en cada cambio de gobierno, las ideologías y organizaciones políticas también cambiaban pero el descontento se mantenía en ascenso, en la era democrática ecuatoriana el voto del ciudadano divaga por varias alternativas y los resultados demostraron que la democracia representativa por sí sola no satisfacía al pueblo, fue cuando la ciudadanía más madura decide en formar organizaciones y movimientos para expresarse en el escenario tradicional del pueblo " las calles", todo esto hizo que en el ciudadano emerja con una profunda desconfianza en los 
partidos políticos. "La pérdida de confianza en los círculos gobernantes ha sido nítidamente sancionada en las urnas." (Ramírez, 2001:234). Lo mencionado anteriormente conjuga con el pensamiento de Boaventura de Sousa Santos (2014):

"Sin la participación más densa y comprometida de los ciudadanos y de las comunidades en la dirección de la vida política, la democracia continuará siendo rehén de la antidemocracia esto es de intereses que generan las mayorías parlamentarias a su favor y en contra de la mayoría de los ciudadanos" (127).

La participación ciudadana entonces nace como recurso oasis, llamado a fortalecer y profundizar la democracia ecuatoriana. Para el 2006 aparece un actor clave en el proceso de transformación democrática del país, el entonces candidato Rafael Correa Delgado quien entendió el malestar ciudadano y lo llevo a un escenario de causa-efecto convirtiéndolo en presidente de la República, ya en el poder cumple con su oferta de campaña la nueva Constitución del 2008 que "gracias a la participación de numerosos sectores organizados de esa población mayoritaria y al contexto de oportunidad política pudo intervenir e incidir efectivamente en la producción de ese documento." (Unda, 2015:298), a partir de ella se establecen sus dos Planes Nacionales de Desarrollo ( Plan Nacional del Buen Vivir 20092013 y el Plan Nacional 2013-2017) que marcan el rumbo de su gestión y en el tema de participación están contenidos en el objetivo diez (PNBV 213-2017) "Garantizar el acceso a la participación pública” y en el Objetivo uno (PNBV 2013-2017) “Consolidar el Estado Democrático y la construcción del poder popular", estos instrumentos buscan "el fortalecimiento de la sociedad mediante el fomento de la participación política y la construcción de poder popular .. (Secretaria Nacional de Planificación y Desarrollo, 2012:97).

Esta nueva Constitución además trae consigo la transversalización de la Participación Ciudadana en el Ecuador y a partir de ella la conformación de la Función de Transparencia y Control Social la creación del Consejo de Participación Ciudadana y Control Social (CPCCS), así como sus mecanismos, temática que se analiza en el punto dos de este documento.

La participación popular se viene a partir de un entendimiento social que el Estado no es suficiente para resolver asuntos públicos, se acepta un esfuerzo en conjunto, una participación ciudadana reconoce al Estado y no trata deliberadamente de aislar sus argumentos sino compartirlos en función del bien común. La democracia participativa carece de ideologías no es ni de izquierda, de centro, ni de derecha, solo debe adaptarse al sistema democrático y a la democracia en sí.

\section{II) Espacios y Mecanismos orientadores de la Participación Ciudadana en el Ecuador a partir de la Constitución del 2008.}


Ramírez Gallegos (2011) en Estado del país menciona, "la nueva Carta Magna otorga un peso sin precedentes a la participación ciudadana en el proceso político y en la vida pública del país. Así de entre los nueve títulos que contiene el texto, uno está enteramente dedicado a la cuestión" (231), de la misma manera varios autores como Noguera (2008), Morales (2008), Ramirez (2010), Cañas \& Birck (2011) consideran que la nueva constitución tiene una naturaleza participativa y que su labor es dotar al ciudadano de un nuevo derecho "la participación" y que su función es profundizar a la democracia; antes en la anterior Constitución constaba el concepto de "medios democráticos" ahora "formas de participación directa", aquí se abre el camino para el establecimiento de los mecanismos de participación. Más adelante en el artículo 95 de la Constitución, dota de especificidad, apertura la inclusión de actores, ámbitos de acción, principios en su ejercicio, modalidades democráticas, pero sobretodo la asignación al ciudadano de un derecho "la participación" que al parecer pocas veces la ejerce, la desconoce, desconfía o no es de su interés.

Pero ahora ¿cuáles son los mecanismos, dispositivos e instituciones que existen y operan dentro de la legislación ecuatoriana?, para resolver esta cuestión se inicia realizando una búsqueda en la legislación vigente tomado en cuenta la Constitución del Ecuador del 2008, La Ley de Participación Ciudadana y la Ley del Consejo de Participación Ciudadana y Control Social a fin de construir una clasificación general de los mecanismos de participación ciudadana en el Ecuador. En la actual legislación mencionada se detecta tres grandes grupos de mecanismos de participación ciudadana por su naturaleza: Mecanismos de participación por medio de la Democracia Directa, Mecanismos de participación en la gestión pública y mecanismos de participación para el control social y gestión pública, esta clasificación a su vez se subdivide como se detalla en el gráfico a continuación:

\section{Gráfico 1. Mecanismos de participación ciudadana en el Ecuador}

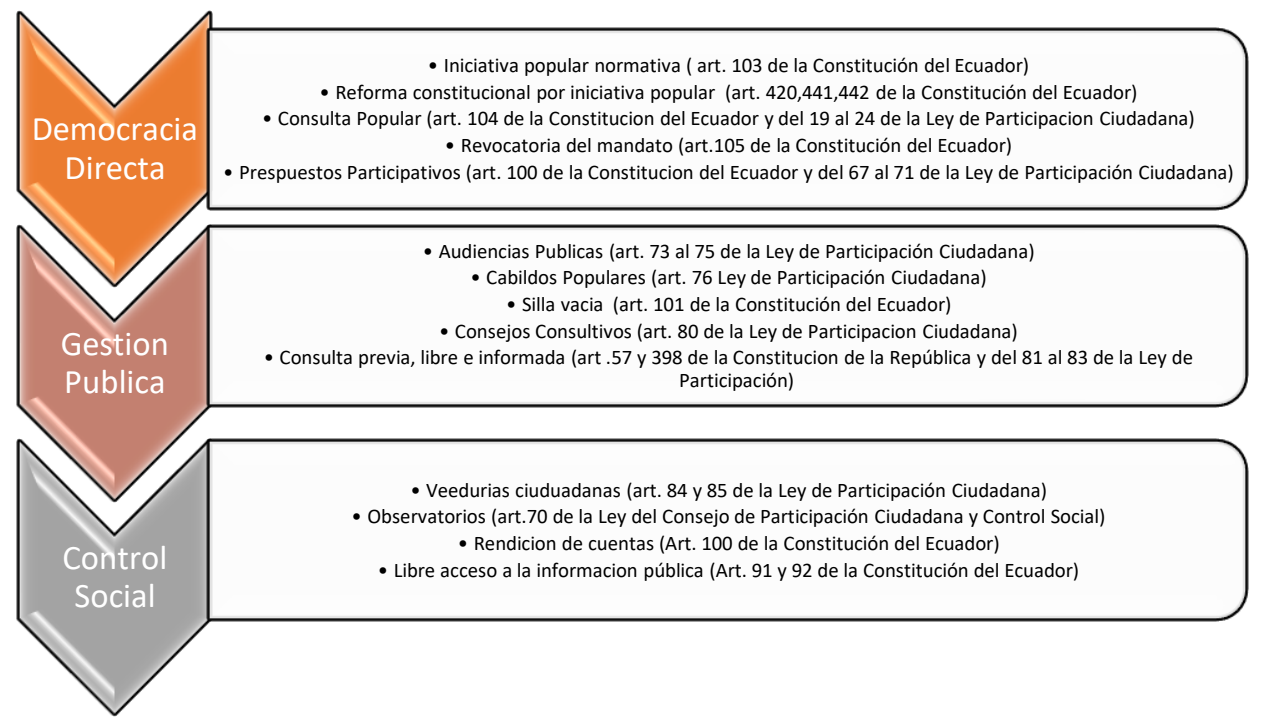

Fuente: Constitución del Ecuador 2008, Ley de Participación ciudadana, Ley del Consejo de Participación Ciudadana y Control Social, (Cañas \& Birck, 2011: 94-113)

Elaborado por: Los autores 
La Constitución vigente en el Ecuador, pasando por dos leyes orgánicas (la Ley Orgánica del Consejo de Participación Ciudadana y la Ley Orgánica de Participación Ciudadana) y finalmente por un Código Orgánico (Código Orgánico de Organización Territorial Autonomía Descentralización), se constituye el marco jurídico que respalda el funcionamiento de la participación ciudadana en el país, cada uno de estos instrumentos como se observó en el cuadro No. 1 tiene su fundamento jurídico estos espacios, mecanismos y dispositivos permiten al ciudadano una amplia gama de oportunidades de participación, que los incentiva desde el ejecutivo, desde los Gobiernos Autónomos Descentralizados y desde la ciudadanía mismo, ahora es menester del soberano en utilizarlos. "Las nuevas herramientas introducidas en la Carta Magna solo pueden generar efectos e impactos en la medida en que la ciudadanía haga uso de ellas. Aún más, es necesario que se utilicen estos mecanismos para que no se conviertan en dispositivos principalmente promovidos por los poderes constituidos:.. " (Cañas \& Birck, 2011:95)

Dentro del Gobierno Central y sus instituciones están innovaciones e iniciativas de participación ciudadana, así lo menciona Acurio (2010) cuando cita a Molina:

"Entre otros mecanismos de interrelación Estado-sociedad, tenemos mesas de dialogo, elaboración participativa de leyes y normativas, convocatorias de gobiernos locales, consultas ciudadanas, foros de discusión sobre políticas sectoriales, gabinetes itinerantes, redes mixtas de actores públicos y privados, procesos de planificación participativa y consejos consultivos. Todas estas iniciativas están orientadas a una nueva democracia y nuevas formas de relación entre la ciudadanía, las organizaciones y la institucionalidad del Estado". (121)

Esta descripción de los mecanismos de participación ciudadana que están planteados dentro de la estructura del Ejecutivo, correlaciona e instrumentaliza lo plasmado en el Plan Nacional de Buen Vivir, además de ellos se logró identificar otras acciones ciudadanas que son consideradas como participación ciudadana y que demuestran su transversalidad, incluso lo contemplado en el COOTAD.

Tabla 1. Otros ejemplos de espacios de participación ciudadana.

\begin{tabular}{|c|c|c|c|}
\hline Legislación & $\begin{array}{c}\text { Objetivo del espacio de } \\
\text { participación }\end{array}$ & $\begin{array}{l}\text { Entidad donde } \\
\text { opera }\end{array}$ & Acción ciudadana \\
\hline $\begin{array}{l}\text { Art. } 170 \\
\text { Constitución }\end{array}$ & $\begin{array}{l}\text { Establecer como uno de los } \\
\text { criterio de selección a la } \\
\text { participación ciudadana }\end{array}$ & Función Judicial & $\begin{array}{l}\text {-Impugnación } \\
\text { ciudadana }\end{array}$ \\
\hline $\begin{array}{l}\text { Art. } 347 \text { y } 359 \\
\text { Constitución }\end{array}$ & $\begin{array}{l}\text { Determinar la participación de } \\
\text { ciudadanos, padres de familia, } \\
\text { estudiantes y docentes en los } \\
\text { sistemas de salud y educación }\end{array}$ & $\begin{array}{l}\text { Sistema Nacional } \\
\text { de Educación }\end{array}$ & $\begin{array}{l}\text {-Plan Decenal de } \\
\text { Educación } \\
\text {-Plan Nacional de } \\
\text { Salud }\end{array}$ \\
\hline
\end{tabular}




\begin{tabular}{|c|c|c|c|}
\hline & & $\begin{array}{l}\text { Sistema Nacional } \\
\text { de Salud }\end{array}$ & \\
\hline $\begin{array}{l}\text { Art. } 39 \text { y } 48 \\
\text { Constitución }\end{array}$ & $\begin{array}{c}\text { Facilitar la participación } \\
\text { política y social de jóvenes y } \\
\text { grupos vulnerables }\end{array}$ & $\begin{array}{l}\text { Instituciones } \\
\text { públicas y } \\
\text { privadas relación } \\
\text { al empleo }\end{array}$ & 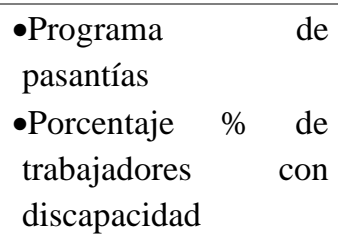 \\
\hline $\begin{array}{l}\text { Art. } 306 \text { y } 308 \\
\text { COOTAD }\end{array}$ & $\begin{array}{l}\text { Reconocer a los barrios y } \\
\text { parroquias urbanas como } \\
\text { unidades básicas de } \\
\text { participación ciudadana }\end{array}$ & $\begin{array}{l}\text { Circunscripción } \\
\text { territorial local }\end{array}$ & $\begin{array}{l}\text {-Barrios, Parroquias } \\
\text { Urbanas } \\
\text {-Comunas, } \\
\text { Comunidades } \\
\text { Recintos }\end{array}$ \\
\hline
\end{tabular}

Fuente: (Asamblea Nacional Constituyente, 2008), (Asamblea Nacional, 2010), (Gobiernos Autonomos Descentralizados; Ministerio de la Coordinación de la Política;, 2011)

Elaborado por: Los autores

En la parte institucional la participación ciudadana puede desenvolverse en dos ámbitos a nivel nacional y local, para este estudio por su importancia en cuanto a su conformación y temas que abordan de interés general describo las instituciones del ámbito nacional, ellos son en los Consejos Nacionales para la Igualdad (art.156 y 157 de la Constitución del Ecuador, art.47 de la Ley de Participación Ciudadana), el Consejo Nacional de Planificación (Art. 279 de la Constitución del Ecuador, art. 48 y 49 de la Ley de Participación Ciudadana), los Consejos Ciudadanos Sectoriales (52 al 55 de la Ley de Participación Ciudadana), y la Asamblea Ciudadana Plurinacional e Intercultural para el Buen Vivir (art. 48 de la Ley de Participación Ciudadana), estas instituciones son de carácter mixtas es decir participan el Estado y la sociedad, convirtiéndose en espacios deliberativos en donde entran en debate temas de la "agenda estatal", es decir se tratan puntos que previamente están diseñados, si bien es cierto son espacios que puede interactuar la ciudadanía, también lo hacen expertos, la mayoría de estos espacios intervienen delegados del ejecutivo e incluso está declarada la presidencia como en el caso del Consejo Nacional para la Igualdad para su representante.

En cuanto a la participación ciudadana en la gestión pública hay opiniones contrarias a la intervención del ciudadano en la gestión pública por ejemplo para Goldfrank (2015) "los partidos políticos opuestos al gobierno de turno y especialmente sus consejales municipales suelen insistir en que las decisiones públicas deben incumbir a los representantes electos, no a una media docena de voluntarios" (107), tambien relata que las decisiones publicas presupuestarias son técnicas y que la participación ciudadana solo estorba y enlentece; Pogrebinschi (2012) propone "que no existe necesariamente una tensión antagónica entre participación y representacion. Las innovaciones democráticas aumentan la participación 
ciudadana directa, pero eso no quiere decir que las instituciones políticas sean menos aptas para representar a los ciudadanos"(p.79-80).

Los mecanismos actuales como las audiencias públicas, cabildos populares, silla vacía, consejos consultivos y consulta previa libre e informada son dispositivos de participacion ciudadana en la gestión pública contenidos en la legislación actual, que pretenden incluir en los procesos de politicas publicas al ciudadano.

\section{Conclusiones.}

- En América Latina y en el Ecuador la democracia participativa a pesar de entes contrarios es un complemento para la democracia representativa, este nuevo esquema busca fortalecer la costumbre democrática de la representación y también revitaliza la esperanza del ciudadano en ser parte de activa y permanente de las decisiones de los entes políticos, gubernativos y burocráticos, la democracia participativa en efecto proporciona espacios de deliberación.

- La participación de las agrupaciones sociales, movimientos indígenas y grupos minoritarios con tendencias ecológicas y otros, fue trascendental para que en el Ecuador y en varios países de América de Sur se consolide la tendencia de la democracia participativa por medio de la participación ciudadana que nació de la inconformidad de la exclusión y hastío de la corrupción.

- La legislación ecuatoriana contiene de manera transversal la participación ciudadana en varios instrumentos, la incluyen en las entidades del gobierno central, en los gobiernos autónomos descentralizados y reconoce la agrupación social en la sociedad.

\section{Referencias bibliográficas}

Acurio, J. S. (2010). Participación en la institucionalidad del Estado. En SENPLADES, Tendencias de la Participación Ciudadana en el Ecuador (págs. 118-136). Quito: El Conejo.

Asamblea Nacional. (2009). Ley Organica del Consejo de Participación Ciudadana y Control Social. Quito.

Asamblea Nacional. (2010). Ley Organica de Participación Ciudadana. Quito: Lexis.

Asamblea Nacional Constituyente. (1998). Constitución Política de la República del Ecuador. Quito: Editorial Juridica del Ecuador.

Asamblea Nacional Constituyente. (2008). Constitución Política de la República del Ecuador. Quito: Lexis.

Bernal, C. (2006). Metodología de la Investigación. México: Pearson Educación.

Cañas, V., \& Birck, N. (2011). Participación Ciudadana y Control Social. Quito: IAEN. 
CLAD. (2009). Carta Iberoamericana de Participación Ciudadana en la Gestión Pública. XI Conferencia Iberoamericana de Ministros de Administración Pública y Reforma del Estado, (págs. 1-15). Lisboa.

Cunill Grau, N. (1997). Repensando lo público a través de la sociedad: nuevas formas de gestión pública y representación social. Caracas: CLAD.

De Sousa Santos, B. (2003). Democracia y Participación; el ejemplo del presupuesto participativo. Madrid: Ediciones de Intervención Cultural /el Viejo Topo.

De Sousa Santos, B. (2014). Democracia al Borde del Caos: ensayo contra la autoflagelación. Bogota: Siglo XXI Editores.

Gobiernos Autonomos Descentralizados; Ministerio de la Coordinación de la Política;. (Febrero de 2011). Codigo Orgánico de Organización Territorial Autonomía y Descentralización. Obtenido de http://www.ame.gob.ec/ame/pdf/cootad_2012.pdf

Goldfrank, B. (2015). Democracia participativa e izquierdas: logros, contradicciones y desafíos. En C. Mouffe, DEMOCRACIA PARTICIPATIVA e IZQUIERDAS: logros, contradicciones y desafios. (págs. 95-120). Quito: Friederich Ebert Stiftung (FES-ILDIS).

Noguera Fernandez, A. (2008). Participación, Función Electoral y Función de Control y Transparencia Social. En R. Avila Santamaría, A. Grijalva Jiménez \& \& R. Martinez Dalmau, Desafio constitucionales. La Constitución ecuatoriana 2008 en perspectiva (págs. 134-157). Quito: V\&M Graficas.

Pogrebinschi, T. (2012). Participación como representación:generación democrática de politicas públicas en Brasil. En M. Cameron, E. Hershberg, \& K. Sharpe, Nuevas instituciones de democracia participativa en America Latina:la voz y sus consecuencias (págs. 77-104). Mexico: FLACSO México.

Ramirez, f., \& Welp, Y. (2011). Nuevas Instituciones participativas y democráticas en América Latina. Iconos, 11-20.

Ramirez, Franklin. (2011). Participación,desconfianza política y Transformación Estatal. La innovación participativa en la Constitución ecuatoriana 2008. En E. d. País, Informe cero. Ecuador 1950-2010, (págs. 232-246). Quito: Activa.

Secretaria Nacional de Planificación y Desarrollo. (2008). Plan Nacional de Desarrollo para el Buen Vivir, 2009-2013 . Quito: SENPLADES.

Secretaria Nacional de Planificación y Desarrollo. (2012). Plan Nacional de Desarrollo para el Buen Vivir 2013-2017. Quito: SENPLADES. 
SENPLADES. (s.f.). Tendencias de la Participación Ciudadana en el Ecuador. Quito: El Conejo.

Unda, R. (2015). Participación política de jóvenes en Ecuador:entre la espiral del desencanto y el desafío del re-encantamiento en torno de la Revolución Ciudadana. En A. Minaert, \& G. Endara, Democracia Participativa e Izquierdas: logros contradicciones y desafios (págs. 295-316). Quito: Friedrich Ebert Stiftung ILDIS. 


\section{PARA CITAR EL ARTÍCULO INDEXADO.}

Carrión Torres, M., Contento Armijos, D., \& Pazmiño Garzón, D. (2019). Democracia y participación ciudadana en el Ecuador. ¿Un espejismo?. Ciencia Digital, 3(3.4.), 5-18. https://doi.org/10.33262/cienciadigital.v3i3.4.829

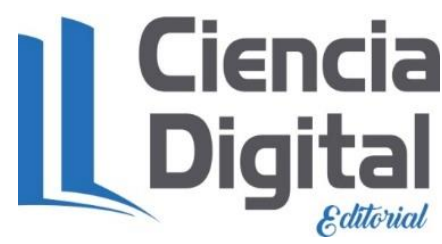

El artículo que se publica es de exclusiva responsabilidad de los autores y no necesariamente reflejan el pensamiento de la Revista Ciencia Digital.

El artículo queda en propiedad de la revista y, por tanto, su publicación parcial y/o total en otro medio tiene que ser autorizado por el director de la Revista Ciencia Digital.
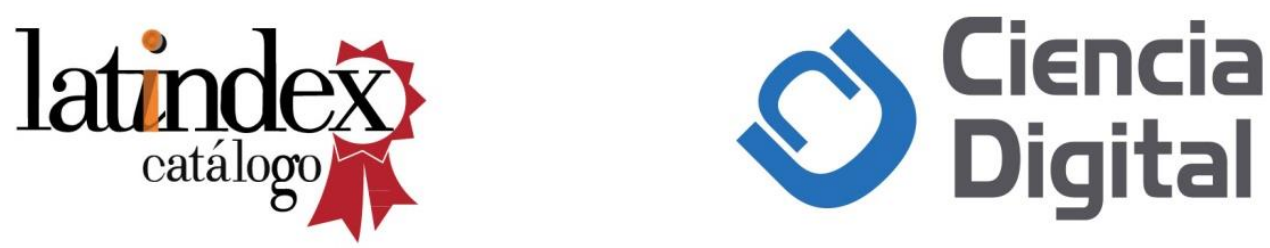